University of Nebraska - Lincoln

DigitalCommons@University of Nebraska - Lincoln

$1-2012$

\title{
A Kinematic Description of the Temporal Characteristics of Jaw Motion for Early Chewing: Preliminary Findings
}

\author{
Erin M. Wilson \\ University of Wisconsin-Madison, emhillman@wisc.edu \\ Jordan R. Green \\ University of Nebraska-Lincoln, jgreen4@unl.edu \\ Gary Weismer \\ University of Wisconsin - Madison, gweismer@wisc.edu
}

Follow this and additional works at: https://digitalcommons.unl.edu/specedfacpub

Part of the Special Education and Teaching Commons

Wilson, Erin M.; Green, Jordan R.; and Weismer, Gary, "A Kinematic Description of the Temporal Characteristics of Jaw Motion for Early Chewing: Preliminary Findings" (2012). Special Education and Communication Disorders Faculty Publications. 71.

https://digitalcommons.unl.edu/specedfacpub/71

This Article is brought to you for free and open access by the Department of Special Education and Communication Disorders at DigitalCommons@University of Nebraska - Lincoln. It has been accepted for inclusion in Special Education and Communication Disorders Faculty Publications by an authorized administrator of DigitalCommons@University of Nebraska - Lincoln. 


\title{
A Kinematic Description of the Temporal Characteristics of Jaw Motion for Early Chewing: Preliminary Findings
}

\author{
Erin M. Wilson, ${ }^{1}$ Jordan R. Green, ${ }^{2}$ and Gary Weismer ${ }^{3}$ \\ 1. Waisman Center, University of Wisconsin-Madison \\ 2. University of Nebraska-Lincoln \\ 3. University of Wisconsin-Madison \\ Corresponding author - Erin M. Wilson, emhillman@wisc.edu
}

\begin{abstract}
Purpose: The purpose of this investigation was to describe age- and consistency-related changes in the temporal characteristics of chewing in typically developing children between the ages of 4 and 35 months and adults using high-resolution optically based motion capture technology.

Method: Data were collected from 60 participants (48 children, 12 adults) across 5 age ranges (beginners, 7 months, 12 months, 35 months, and adults); each age group included 12 participants. Three different food consistencies were trialed as appropriate. The data were analyzed to assess changes in chewing rate, chewing sequence duration, and estimated number of chewing cycles.

Results: The results revealed both age- and consistency-related changes in chewing rate, sequence duration, and estimated number of chewing cycles, with consistency differences affecting masticatory timing in children as young as 7 months of age. Chewing rate varied as a function of age and consistency, and chewing sequence duration was shorter for adults than for children regardless of consistency type. In addition, the results from the estimated number of chewing cycles measure suggest that chewing effectiveness increased with age; this measure was also dependent on consistency type.

Conclusions: The findings suggest that the different temporal chewing variables follow distinct developmental trajectories and are consistency dependent in children as young as 7 months of age. Clinical implications are detailed.
\end{abstract}

Keywords: chewing, kinematics, development, consistency, timing

S uccessful oral feeding depends on the temporal coordination of multiple oral and pharyngeal structures. The coordination for suckling, one of the earliest-appearing oral-feeding behaviors, seems to be relatively well established at birth (Finan \& Barlow, 1998). For example, Bosma (1986) described oral and pharyngeal movements during the early stages of suckling development as already rhythmic, a suggestion that has been confirmed by a number of investigators (Arvedson, Rogers, \& Brodsky, 1993; Morris \& Klein, 2000). Morris and Klein suggest that "rhythm is the most consistent characteristic of feeding patterns during the first 3 months of life" (2000, p. 67). These descriptions might be interpreted to suggest that the basic temporal organization of feeding patterns is relatively well established in early infancy.

Although the basic rhythmical structure of oral movements during feeding may be well established during infancy, empirically derived knowledge about the development of mandibular control for early chewing is very limited. To date, only a small number of quantitative studies have been conducted (Ahlgren, 1966; Gisel, 1988, 1991; Green et al., 1997; Schwaab, Niman, \& Gisel, 1986; Schwartz, Niman, \& Gisel, 1984; Steeve, Moore, Green, Reilly, \& Ruark McMurtrey, 2008), and even fewer investigations have targeted chewing at its earliest developmental stages (Gisel, 1991; Steeve et al., 2008; Steeve \& Moore, 2009; Wilson \& Green, 2009). Moreover, many of these studies have not accounted for the developmental progression in food consistency (i.e., progression from soft to hard food); in contrast, the significant effects of bolus consistency on chewing rate is well documented in the adult literature (Anderson, Throckmorton, Buschang, \& Hayasaki, 2002; Arizumi, 1989; Filipic \& Keros, 2002; Horio \& Kawamura, 1989; Karkazis, 2002; 
Karkazis \& Kossioni, 1997, 1998; Lundeen \& Gibbs, 1982; Mioche \& Peyron, 1995; Peyron \& Mioche, 1994; Peyron, Mioche, \& Culioli, 1994; Peyron, Mioche, Renon, \& Abouelkaram, 1996; Steiner, Michman, \& Litman, 1974). Gisel and colleagues reported that chewing timing is also affected by bolus consistency in children (Gisel, 1988; Schwaab et al., 1986; Schwartz et al., 1984) even as young as 6 months of age (Gisel, 1991). Therefore, an improved understanding of chewing development will require consistency-specific descriptions of age-related changes in mandibular control. Ideally, these descriptions will begin with the earliest stages of chewing to describe the transition from primitive munching to mature chewing (Morris \& Klein, 2000).

\section{Chewing Rate}

The frequency of jaw oscillation during chewing (i.e., chewing rate) is one of the few variables that has been studied during development (Ahlgren, 1966; Gisel, 1988; Green et al., 1997; Schwaab et al., 1986; Schwartz et al., 1984; Sheppard \& Mysak, 1984; Steeve et al., 2008). The findings from this research for both children and adults are summarized in Table 1. Despite differences across investigations in participant ages, bolus consistencies, and methodologies, the findings are remarkably similar, suggesting that variation in chewing rate across age is relatively small. Will kinematic representations of chewing-like behavior yield similar results?

\section{Chewing Sequence Duration}

Chewing sequence duration is commonly reported in the pediatric literature and provides normative information about the amount of time required to manipulate a bolus in preparation for swallow; however, the findings from studies on age-related changes in the duration of chewing sequences (i.e., the amount of time required to break down a bolus) have been mixed. Sheppard and Mysak (1984) observed an increase in chewing duration with age in young infants (age $\leq 35$ weeks), whereas Gisel and colleagues reported a decrease in chewing duration in children from 6 months to 2 years of age (Gisel, 1991), from 2 to 5 years of age (Schwaab et al., 1986), and from 2 to 8 years of age (Gisel, 1988); however, there was no significant difference in chewing duration in children between 4 and 5 years of age (Schwartz et al., 1984).

\section{Chewing Effectiveness}

Temporal measures of chewing have also been used to document changes in chewing effectiveness with age. For example, Gisel $(1988,1991)$ reported a decrease in both the chewing sequence duration and the
Table 1. Chewing rates from previous investigations.

\begin{tabular}{lll}
\hline Age group & Lead author (year) & Chewing rate \\
\hline Adults & Möller (1966) & $1.46-1.73 \mathrm{~Hz}$ \\
Adults & Steiner et al. (1974) & $0.60-1.80 \mathrm{~Hz}$ \\
Children & Ahlgren (1966) & $1.73 \mathrm{~Hz}$ \\
Children & Schwartz & $0.8 \pm 0.2-$ \\
& $\quad$ et al. (1984) & $1.3 \pm 0.5 \mathrm{~s} /$ cycle \\
Children & Sheppard & \\
& $\quad$ and Mysak (1984) & $0.36-1.1 \mathrm{~Hz}$ \\
Children & Schwaab (1986) & $0.62-1.25 \mathrm{~Hz}$ \\
Children & Gisel (1988) & $0.71-1.25 \mathrm{~Hz}$ \\
Children & Green (1997) & $0.88-2.11 \mathrm{~Hz}$ \\
$\quad$ (12-38 months) & & \\
Children (9 months) & Steeve (2008) & $1.23-1.99 \mathrm{~Hz}$ \\
\hline
\end{tabular}

number of chewing cycles required to chew and swallow a bolus. Results from other investigations, however, suggest that age does not influence measures of chewing effectiveness at certain age intervals (Schwaab et al., 1986; Schwartz et al., 1984). Electromyographic studies of mandibular muscle activation patterns have provided perhaps the strongest evidence of increased chewing effectiveness with age (Green et al., 1997; Steeve et al., 2008). For example, older children exhibit the same chewing frequency as younger children but with shorter bursts of muscle activity (Green et al., 1997).

\section{Relevance to Current Models of Feeding Development: The Role of Central Pattern Penerators}

The oral motor coordination for chewing develops in the context of significant neurologic, neuromotor, and anatomic change. Therefore, kinematic-based observations have the potential to provide new insights into the driving forces for change and/or behavioral stability in chewing performance across age. Many of the current models of feeding assign a primary role to brainstem central pattern generators (CPGs) for regulating coordination among oral muscles for early sucking and chewing (Agrawal \& Lucas, 2002; Barlow \& Estep, 2006; Dellow \& Lund, 1971; Finan \& Barlow, 1996, 1998; Lund, 1991; Lund, Appenteng, \& Seguin, 1982; Lund \& Kolta, 2006). The CPG, which has been confirmed in nonhuman animal models, acts as an internal rhythm regulator and is affected by both peripheral and central input. The CPG sends alternating activation signals to antagonistic muscle pairs to produce the rhythmic jaw-opening and -closing pattern characteristic of chewing (Agrawal \& Lucas, 2002; Lund, 1991). The observation of significant developmental change in the temporal characteristics of chewing pat- 
terns may provide a better understanding of how CPG mechanisms are gradually tuned through experience to accommodate significant developmental changes in chewing anatomy and neuromuscular function. Alternatively, the observation that chewing rate does not change with age might be interpreted to support the robust nature of the masticatory CPG, because, for example, the rate of chewing might be expected to decrease with age as mandibular structures become more massive with growth.

\section{Relevance to Current Therapeutic Approaches}

Some clinical descriptions of disordered feeding have noted irregularities in the temporal characteristics of chewing movements. Consequently, certain early feeding therapies have focused on facilitating the rhythmicity, for example, of early feeding movements (Morris \& Klein, 2000). Because many aspects of chewing coordination change with age, quantitative information about the temporal aspects of early chewing development is needed to establish (a) empirically sound benchmarks for gauging the presence and severity of early feeding disorders and (b) developmentally appropriate therapeutic goals.

\section{Purpose}

A more current account of the development of temporal characteristics for chewing is warranted with the recent advent of methods for noninvasively tracking jaw motion in young children. The aim of this investigation was to describe age-related changes in the temporal characteristics of chewing in typically developing children between the ages of 4 and 35 months and in adults using high-resolution, optically based motion capture technology. The following three aspects of chewing timing were investigated cross-sectionally: (a) chewing rate, (b) chewing sequence duration, and (c) estimated number of chewing cycles. The effects of consistency on the agerelated changes of these variables were also examined.

\section{Method}

\section{Participants}

The investigational protocol was approved by the University of Wisconsin's Institutional Review Board. Following approval, data were collected from 60 participants (48 children, 12 adults) across five age ranges (beginners, 7 months, 12 months, 35 months, and adults); each age group included 12 participants. The age ranges were selected to reflect documented stages in the development of mastication (Arvedson, 1993; Arvedson \& Lefton-Greif, 1996; Arvedson et al., 1993; Bosma, 1986; Morris \& Klein, 2000; Pinder \& Faherty, 1999; Pridham, 1990; Sheppard \& Mysak, 1984). More specifically, findings from this literature suggest that at 7 months of age, the chewing pattern is emerging; at 12 months of age, the basic chewing pattern has been established; and at 35 months, the basic chewing pattern has become considerably refined. Because parents introduce spoon-feeding at a variety of ages, the "beginner" age group comprised children ranging from 4 to 6 months of age, all of whom had approximately 2 weeks of experience with foodstuff prior to the data collection session. It is during this beginner age range that chewing is a novel behavior, and the basic chewing pattern is typically not well established. The data from the adult subjects provided a theoretical end point for jaw performance during chewing.

Participants were judged to be typically developing based on (a) an informal developmental questionnaire administered during an initial telephone call and (b) use of the Ages \& Stages Questionnaire (ASQ) - Second Edition (Squires \& Bricker, 1999), which is a parental report screener that assesses development across five domains, including fine- and gross-motor control, communication, problem-solving, and personal-social skills. Parents were asked to complete the ASQ within one month of completing the data collection session and approximately six months after the session to ensure the participants continued to demonstrate typical development. Data from one beginner participant were excluded from the data corpus because of a developmental delay that was identified within six months of participating.

\section{Materials and Procedure}

Three-dimensional motion capture system. Adults were seated in a chair, and child participants were placed in an infant seat on a chair and secured with lap and shoulder straps. Data were collected using a three-dimensional motion capture system (Vicon, 250). The system consisted of five cameras that registered jaw motion at 60 frames per second and a computer workstation that used the five cameras to derive the three-dimensional position of markers strategically located on the chin during chewing.

Marker system. The small reflective markers (approximately two millimeters) were placed on seven facial landmarks. One marker was placed on the gnathion (JC), two were placed $2 \mathrm{~cm}$ to the right (JR) and left of the gnathion (JL), and a marker array was placed on the forehead (see Figure 1). The forehead marker array defined an anatomically based coordinate system (Wilson \& Green, 2009). Although three markers were placed on the chin to ensure optimal tracking, movement from only one chin marker per chewing trial was selected for 


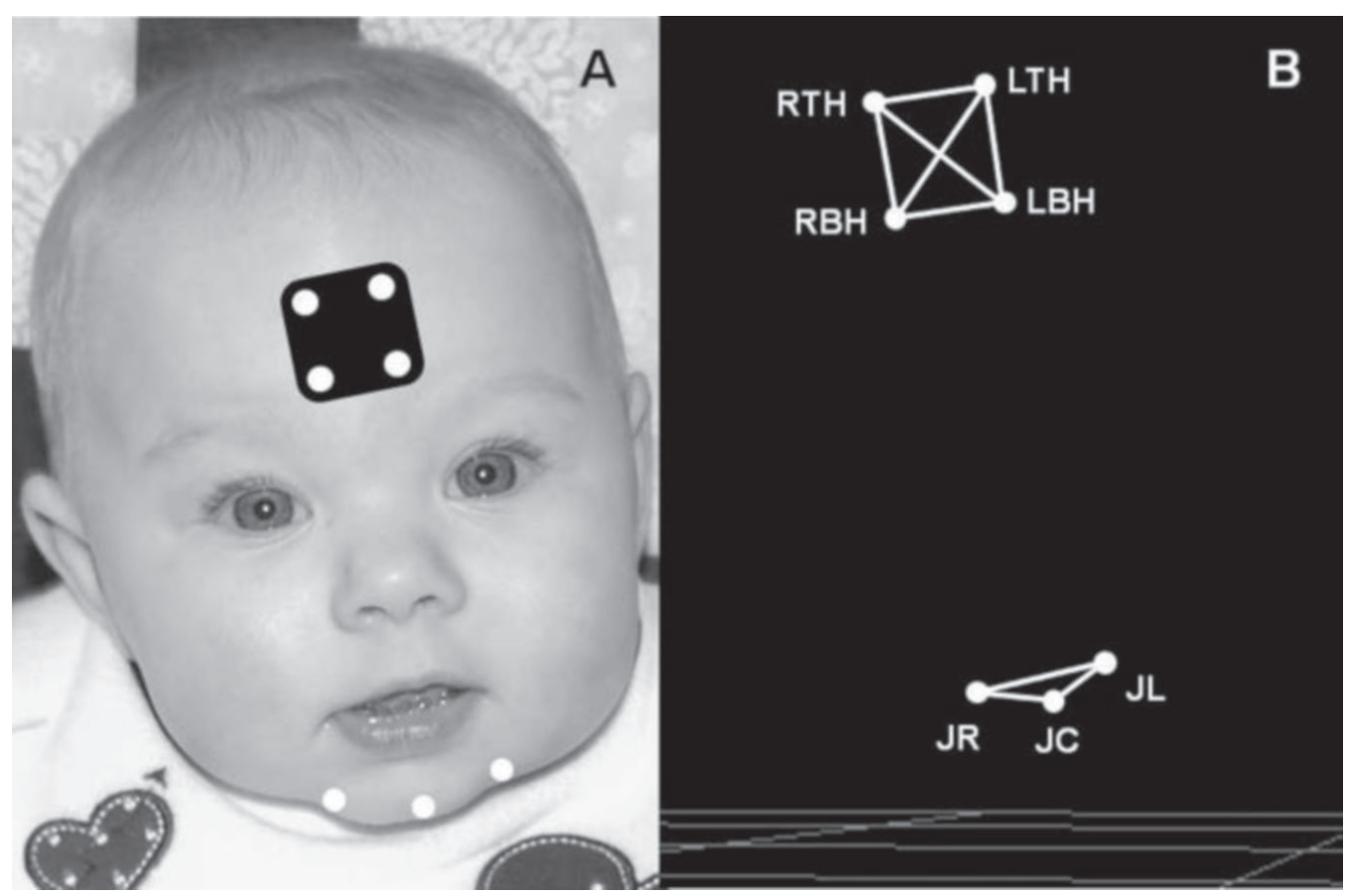

Figure 1. Marker array. Panel A: Marker placement on infant. Panel B: Corresponding marker representation in three-dimensional space.

analysis. Whenever possible, the JL or JR markers were selected for analysis because movement of the markers to the right and left of the gnathion more accurately represent motion of the mandible (Green, Wilson, Wang, \& Moore, 2007). However, timing is well preserved regardless of which marker is selected (Chmielewski, Feine, Maskawi, \& Lund, 1994; Green et al., 2007; Häggman-Henrikson, Eriksson, Nordh, \& Zafar, 1998; Jemt \& Hedgård, 1982; Zafar, Nordh, \& Eriksson, 2002).

Food. Food was provided by the family or investigators. Because food selections and consistencies were based on each child's typical diet, we were unable to administer the same foodstuff to each participant. We therefore required that the food fit into three different food consistency categories: puree, semisolid, and solid. Consistency classification was carefully judged by the primary investigator and was based on the criteria of the National Dysphagia Diet (2002). Because the ability to manage different consistencies is a learned behavior, the participants were asked to trial each consistency only if they had approximately two weeks of experience with a specific consistency classification. The beginner age group, therefore, was only capable of trialing a puree-consistency food. The caregivers and/or primary investigator fed each child, and the size of each food bite was consistent across trials and participants (i.e., 1 teaspoon). The adult participants fed themselves, but bolus size was the same as for the children, and administration was closely monitored by the primary investigator. As appropriate, attempts were made to administer five trials of each consistency type to every participant, although because of developmental level and/or compliance, not every participant accepted all five trials (see Tables 3 \& 4).

Missing data. Missing data occurred if the marker was not captured in view of at least two cameras. Data were only included in the final data corpus if at least $75 \%$ of the chewing sequence was present for one of the jaw markers; $7.01 \%(37 / 528)$ of the sequences in the final corpus had $<25 \%$ missing data. To maximize the yield from the data set for the rate analysis, each file with missing data was further parsed to include the greatest portion of continuous data while excluding the missing segment; however, only complete sequences were analyzed for the measures of chewing sequence duration and estimated number of chewing cycles. Finally, a requirement that each chewing sequence had to contain at least 1.5 cycles of chewing (i.e., jaw at minimal displacement, maximum displacement, minimum displacement, and maximum displacement) was established. This criterion was established to ensure that all sequences contained actual chewing motion because participants were occasionally observed to almost immediately swallow certain consistency boluses. Sixty-one trials were excluded as a result of this criterion (see Table 2).

Data editing. Movement data were parsed into chewing sequences based on the continuous digital video recordings. A chewing sequence began at the point of maximal jaw closure after the spoon had been removed from the mouth and ended approximately at the point 
Table 2. Distribution of chewing sequences across age groups and consistency categories excluded due to lack of chewing motion.

\begin{tabular}{llll}
\hline Age group & Puree & Semisolid & Solid \\
\hline Beginners & 4 & 0 & 0 \\
7-month-olds & 4 & 0 & 0 \\
12-month-olds & 7 & 0 & 0 \\
35-month-olds & 17 & 1 & 0 \\
Adults & 28 & 0 & 0 \\
Total number of trials & 60 & 1 & 0 \\
\hline
\end{tabular}

lip pursing and/or laryngeal motion was observed for the swallow. ${ }^{1}$ The onset and offset frame for each event was identified by the primary investigator after rigorous inspection of the video data following rule-based criteria. After this initial parsing of the data files, the chewing sequences were trimmed further to eliminate movement related to initial bolus positioning, clearing of the gums/teeth prior to the swallow, and the swallow. The additional trimming was accomplished by parsing the mid- $90 \%$ of the sequence for puree-consistency trials and mid- $80 \%$ for semisolid- and solid-consistency trials. See Figure 2.

Data filtering. Prior to analysis, the movement signals were digitally low-pass filtered $\left(f_{\mathrm{lp}}=10 \mathrm{~Hz}\right)$ using a zero-phase shift forward and reverse digital filter (Butterworth, 8 pole). Each signal was subjected to high-pass filtering $\left(f_{\mathrm{hp}}=0.20 \mathrm{~Hz}\right)$ to remove high-amplitude, low-frequency components of the displacement signals. High-pass filtering was necessary because the average position of the mandible elevates with bolus breakdown; if the data were not high-pass filtered, this low-frequency component of the chewing signal would dominate the frequency spectrum, obscuring the higherfrequency component related to the frequency of chew.

\section{Analysis Procedures}

Analysis 1: Age- and consistency-related changes in chewing rate. The jaw movement signals were analyzed to determine how the rate of chewing changed with age and varied across food-consistency categories. The rate of jaw movement was computed algorithmically by performing fast Fourier transforms (FFT) on the vertical jaw displacement signals. This analysis yielded a spectrum associated with each chewing sequence from which the most prominent peak in the spectrum was recorded.

1. Jaw motion for infant chewing is highly variable at times making the end point of the chewing sequence difficult to detect. Therefore, to maximize reliability of the parsing process, parsing rules were developed to ensure that the end point of the sequence was consistent across all sequences regardless of age. Based on a large number of observations, the most reliable indication of the end of a chewing sequence (from the video observation of the behavior) was unquestionably the swallow in the infant participants.

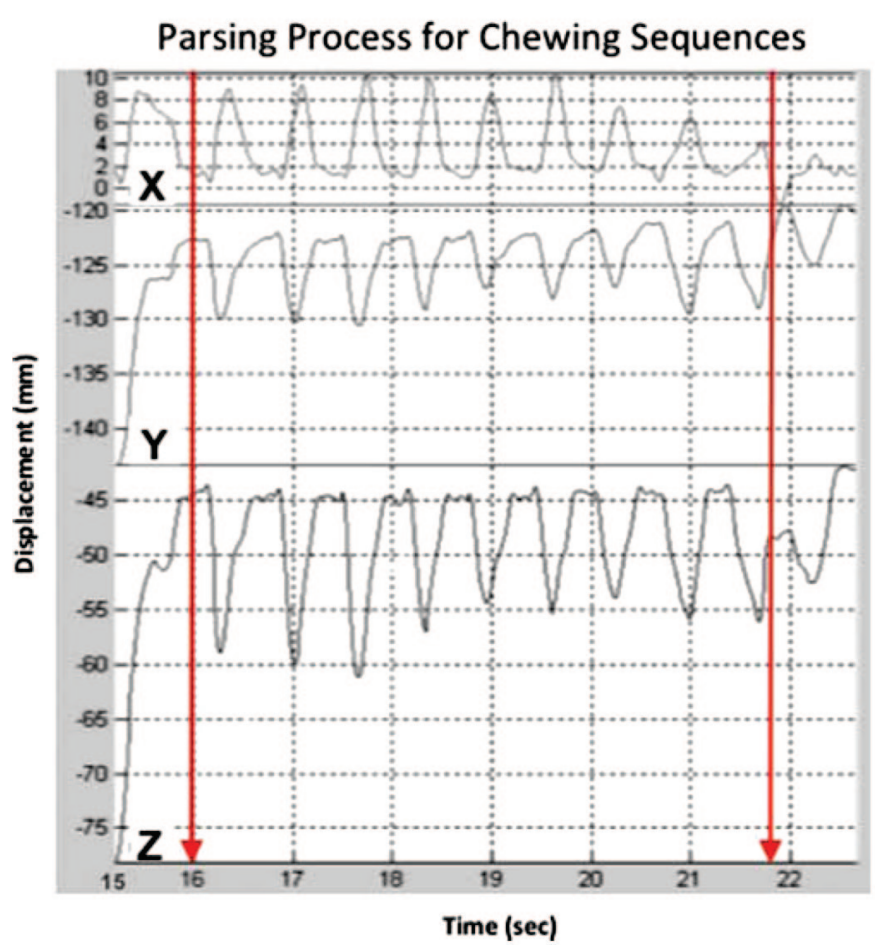

Figure 2. Parsing process for chewing sequences. This figure illustrates the time history of a chewing sequence. Panels $X, Y$, and $Z$ represent jaw movement in the horizontal, vertical, and anterior-posterior dimensions, respectively. The chewing sequence represented was parsed at the point of maximal jaw closure after the spoon had been removed from the mouth and ended approximately at the point laryngeal motion was observed for the swallow. The downward-facing arrows mark the mid- $80 \%$ of the sequence, which was selected in the second phase of the parsing process. The portion of the sequence between the arrows is what was analyzed for each analysis. Note how the segments outside of the arrows, not consistent with the primary motion of the chewing sequence, were excluded because of the two-phase approach to parsing in this investigation.

Analysis 2: Age- and consistency-related changes in chewing sequence duration. Age- and consistency-related changes on the duration of each chewing sequence were determined by measuring the duration (in seconds) of each parsed chewing sequence (i.e., mid-80-90\%).

Analysis 3: Age- and consistency-related changes in the estimated number of chewing cycles. An estimate of the number of chewing cycles for each chewing sequence was calculated by multiplying the rate of each chewing sequence $(\mathrm{Hz})$ by the duration of the chewing sequence (i.e., Rate $x$ Duration). The estimated number of cycles provided an indirect measure of chewing effectiveness; that is, more effective chews are assumed to require fewer cycles to break down a bolus than less effective chews. The numbers of chew cycles were estimated 
rather than measured directly from the kinematic traces because individual jaw oscillations during the chewing of real food (vs. gum, for example) are difficult to identify reliably, particularly in the infant participants, based on kinematic landmarks such as peaks and troughs.

\section{Statistical Design}

Because each child was administered multiple trials of each consistency, the data were analyzed statistically using a hierarchical linear model (HLM) as described by Raudenbush and Bryk (2002). Specifically, a two-level HLM model was fit (repeated measures nested within subject) in which consistency-type variables (solid consistency and semisolid consistency) were entered as dummy-coded predictors at Level 1, and age category variables (beginner, 7 months, 12 months, 35 months) were entered as dummy-coded predictors at Level 2. Consequently, in each analysis, the puree-consistency condition and adult age category condition functioned as reference categories. Both the intercept coefficient as well as the slopes attached to the two dummy-coded consistency category variables were treated as random, allowing each child to have a unique effect with respect to each of the three food types. From this model, age effects were tested both (a) across all consistency categories and (b) in relation to differences between specific pairs of consistency categories. The comparisons occurred by setting up specific contrasts (to avoid inflating the Type I error rate), which were tested using chi-square tests (e.g., puree vs. semisolid; semisolid vs. solid). All HLM models as well as contrasts were fit and tested using the HLM 6.0 program (Raudenbush, Bryk, \& Congdon, 2006).

\section{Results}

A total of 528 chewing sequences were evaluated (see Tables 3,4). Descriptive statistics for the analyses are displayed in Figures 3-5.

Table 3. Distribution of chewing sequences across age groups, subjects, and consistency categories for chewing rate.

\begin{tabular}{llll}
\hline Age group & Puree $(n)$ & Semisolid $(n)$ & Solid $(n)$ \\
\hline Beginners & $57(11)$ & $0(0)$ & $0(0)$ \\
7-month-olds & $79(11)$ & $9(2)$ & $8(3)$ \\
12-month-olds & $58(12)$ & $29(9)$ & $30(10)$ \\
35-month-olds & $24(8)$ & $55(12)$ & $38(11)$ \\
Adults & $27(8)$ & $56(12)$ & $58(12)$ \\
Total number of trials & 245 & 149 & 134
\end{tabular}

$n$ represents the number of participants who trialed each consistency within that age group.

\section{Chewing Rate}

Age effects. As depicted in Figure 3, the beginner age group had a slightly slower chewing rate than did the 7-month-old, $\chi^{2}(1, N=58)=4.91, p<.05 ; 12$-monthold, $\chi^{2}(1, N=58)=7.64, p<.01$; and adult, $\chi^{2}(1, N=58)$ $=7.52, p<.01$, age groups for the puree-consistency food. The 12-month-old children chewed the semisolidconsistency food at a slightly slower rate than did the 35 -month-old, $x^{2}(1, N=58)=7.52, p<.01$, and adult, $\chi^{2}(1, N=58)=9.58, p<.01$, age groups. The only significant difference in chewing rate detected within the solid-consistency category was between the 7- and 12-month-old age groups; specifically, the 7-month-old children chewed the solid-consistency food at a significantly faster rate than the 12-month-old children, $\chi^{2}(1, N$ $=58)=3.74, p \leq .05$.

Consistency effects. As depicted in Figure 3, the 7-month-old children chewed the semisolid-consistency food at a significantly slower rate than they did the solid-consistency food, $\chi^{2}(1, N=58)=11.17, p<.01$. Interestingly, no consistency effect was detected for the 12-month-old age group. Within the 35-month-old age group, the children chewed the puree-consistency food at a significantly slower rate than they chewed the semisolid-consistency category, $\chi^{2}(1, N=58)=5.46, p<.05$, a finding that was also detected within the adult age group, $\chi^{2}(1, N=58)=4.71, p<.05$.

\section{Chewing Sequence Duration}

Age effects. The adults chewed puree-consistency food for a significantly shorter amount of time than all of the other age groups: beginner, $\chi^{2}(1, N=58)=13.06, p<.001$; 7-month-old, $\chi^{2}(1, N=58)=10.93, p<.01 ; 12$-monthold, $\chi^{2}(1, N=58)=16.13, p<.001$; and 35-month-old, $\chi^{2}(1, N=58)=14.99, p<.001$ (see Figure 4$)$. The adults also chewed the semisolid-consistency food for a significantly shorter amount of time than all of the other age groups capable of managing that consistency: 7-monthold, $\chi^{2}(1, N=58)=3.33, p=.06 ; 12$-month-old, $\chi^{2}(1, N$

Table 4. Distribution of chewing sequences across age groups, subjects, and consistency categories for chewing sequence duration and estimated number of chewing cycles.

\begin{tabular}{llll}
\hline Age group & Puree $(n)$ & Semisolid $(n)$ & Solid $(n)$ \\
\hline Beginners & $44(11)$ & $0(0)$ & $0(0)$ \\
7-month-olds & $70(11)$ & $7(2)$ & $5(2)$ \\
12-month-olds & $51(12)$ & $24(9)$ & $19(8)$ \\
35-month-olds & $20(8)$ & $47(12)$ & $30(10)$ \\
Adults & $24(8)$ & $54(12)$ & $56(12)$ \\
Total number of trials & 209 & 132 & 110 \\
\hline
\end{tabular}




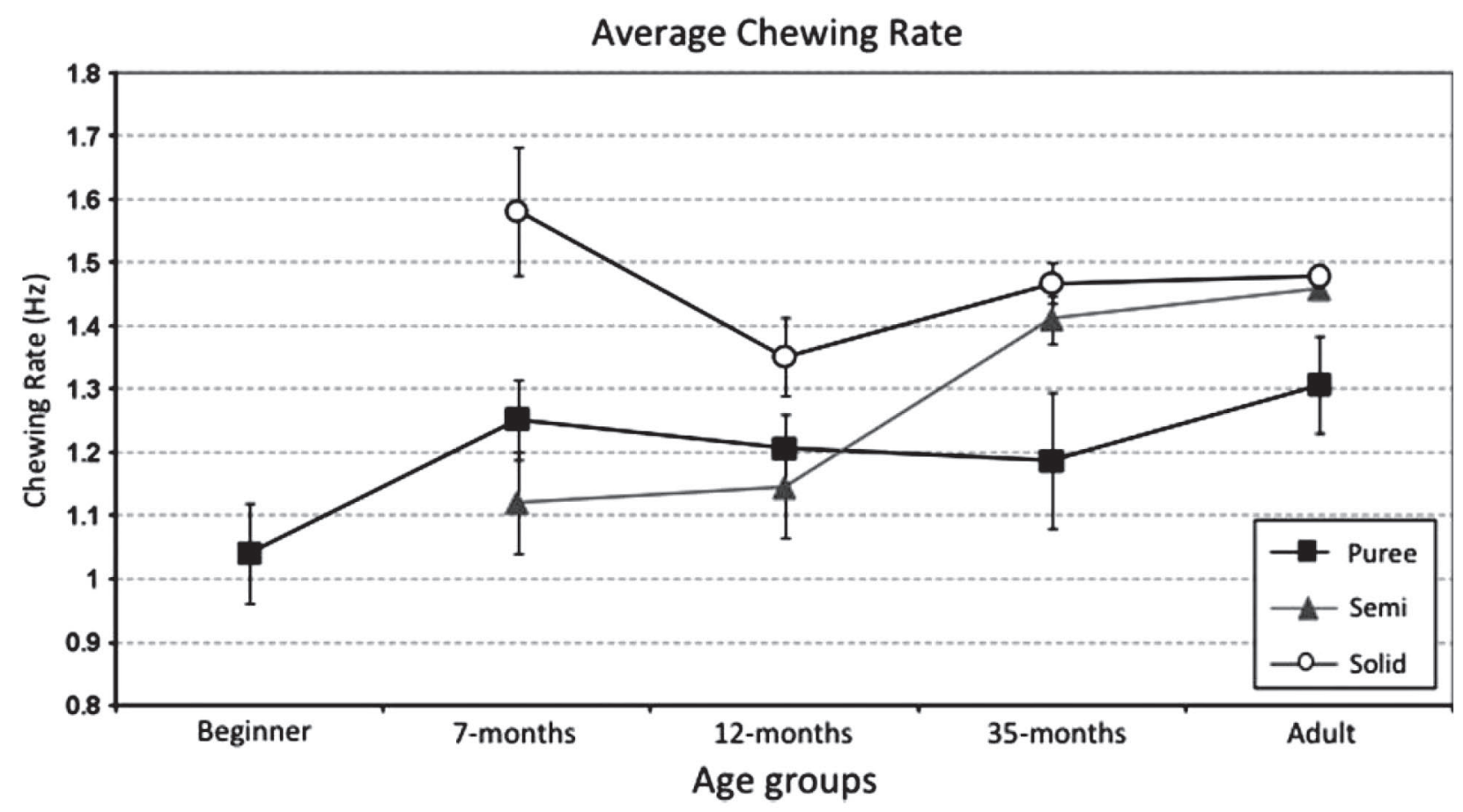

Figure 3. Average chewing rate. This figure illustrates the average chewing rate from beginner to adult participants across all three consistency categories. The error bars represent average standard error across participants.

$=58)=3.74, p=.05 ; 35$-month-old, $\chi^{2}(1, N=58)=4.38$, $p<.05$. Within the solid-consistency category, the duration of the adults' chewing sequences was significantly shorter than that of the 12-month-old, $x^{2}(1, N=58)=$ $4.93, p<.05$, and the 35-month-old, $\chi^{2}(1, N=58)=7.70$, $p<.01$, children. No significant difference was detected between the 7-month-old age group and the adults.

Consistency effects. As seen in Figure 4, the 7-monthold children chewed the puree-consistency food for a significantly shorter amount of time than they did the

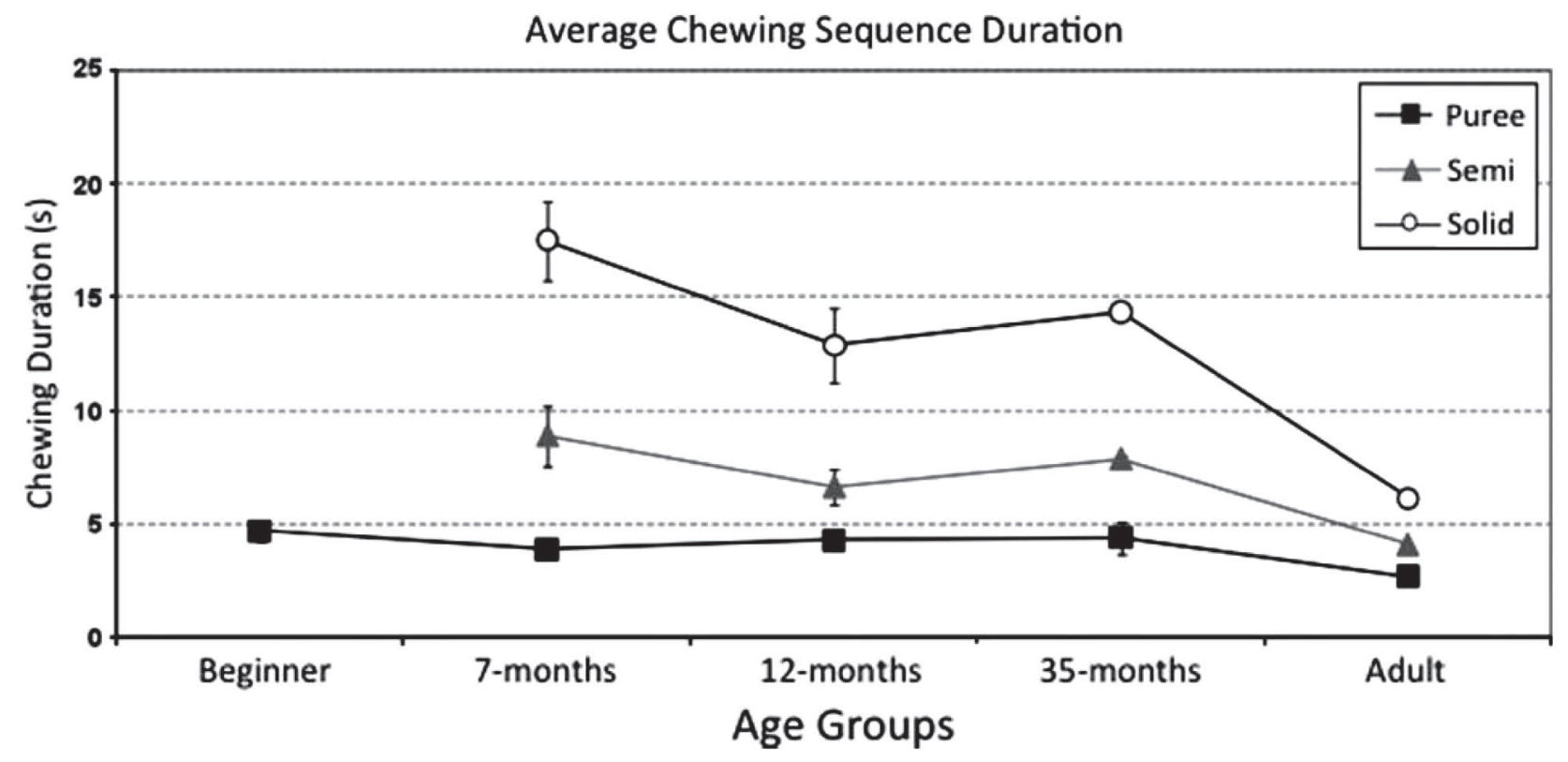

Figure 4. Average chewing sequence duration. This figure illustrates the average chewing sequence duration from beginner to adult participants across all three consistency categories. The error bars represent average standard error across participants. Semi $=$ semi-solid. 


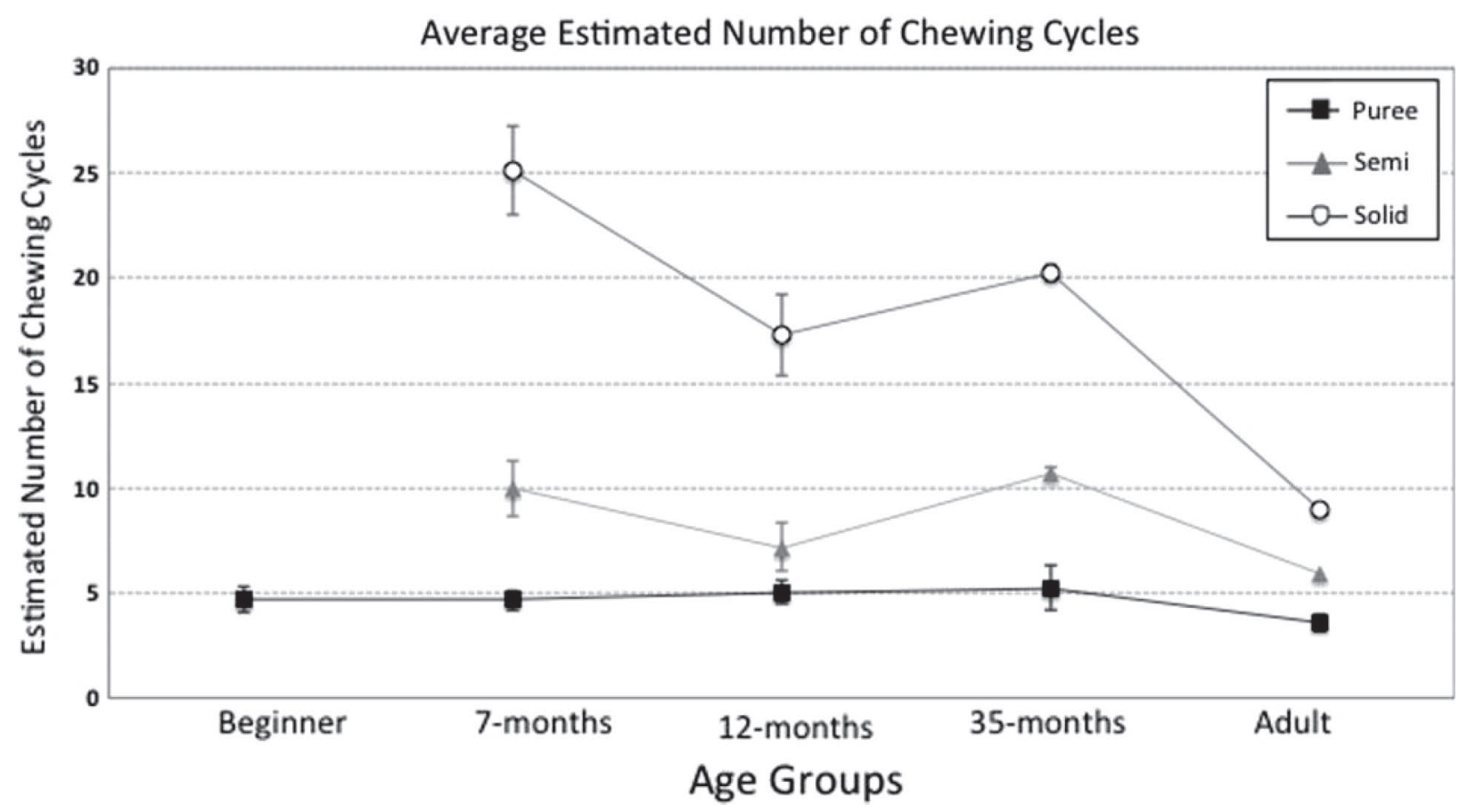

Figure 5. Average estimated number of chewing cycles. This figure illustrates the average estimated number of chewing cycles (Rate x Duration) from beginner to adult participants across all three consistency categories. The error bars represent average standard error across participants.

semisolid-consistency food, $\chi^{2}(1, N=58)=4.39, p<.05$. The 12-month-old children demonstrated longer chewing sequence durations for the solid-consistency food than for the semisolid-consistency food, $x^{2}(1, N=58)=$ $3.21, p=.069$. The 12-month-old children also chewed the semisolid-consistency food for a significantly longer period than they did the puree-consistency food, $\chi^{2}(1, N=58)=3.95, p<.05$. The pattern detected in the 12-month-old age group was also detected in the 35 -month-old and adult age group. That is, all three age groups - the 12-month-olds, $\chi^{2}(1, N=58)=3.95, p<.05$; the 35-month-olds, $\chi^{2}(1, N=58)=3.86, p<.05$; and the adults, $x^{2}(1, N=58)=13.25, p<.001-$ chewed pureeconsistency food for a shorter amount of time than they did semisolid-consistency food. Similarly, the 35-monthold age group, $x^{2}(1, N=58)=3.81, p<.05$, and the adults, $\chi^{2}(1, N=58)=29.92, p<.0001$, chewed the semisolid-consistency food for a shorter amount of time than they chewed the solid-consistency food. However, this finding failed to reach significance for the 12-month-old age group, $\chi^{2}(1, N=58)=3.21, p=.069$.

\section{Estimated Number of Chewing Cycles}

Age effects. The adults had a significantly smaller estimated number of chewing cycles for the puree-consistency food category than any of the other age groups -7 month-olds, $\chi^{2}(1, N=57)=4.50, p<.05 ; 12$-month-olds, $x^{2}(1, N=57)=7.45, p<.01$; and 35-month-olds, $X^{2}(1, N$ $=57)=5.14, p<.05-$ with the exception of the beginner age group, where no significant difference was detected between the two groups (see Figure 5). No significant age effects were detected within the semisolid-consistency food group. Within the solid-consistency food category, the adults had a significantly smaller estimated number of chewing cycles than did the 35-month-old age group, $\chi^{2}(1, N=57)=9.10, p<.01$.

Consistency effects. As depicted in Figure 5, for adults, a smaller estimated number of chewing cycles was observed for puree-consistency food than for semisolidconsistency food, $\chi^{2}(1, N=57)=17.28, p<.001$, and for semisolid-consistency food than for solid-consistency food, $x^{2}(1, N=57)=33.01, p<.00001$. The pattern detected in the adult age group was also detected in the 12 - and 35-month-old age groups. That is, the 12-montholds, $\chi^{2}(1, N=57)=5.54, p<.05$, and the 35-month-olds, $\chi^{2}(1, N=57)=3.64, p=.05$, demonstrated a smaller estimated number of chewing cycles for the puree-consistency food than for the semisolid-consistency food. Similarly, the 12-month-old, $\chi^{2}(1, N=57)=4.83, p<.05$, and 35 -month-old, $x^{2}(1, N=57)=4.88, p<.05$, age groups had a smaller estimated number of chewing cycles for semisolid- than for solid-consistency food.

In contrast, the 7-month-old participants, $\chi^{2}(1, N=$ $57)=4.61, p<.05$, only had a smaller estimated number of chewing cycles for the semisolid consistency than for the solid consistency. There was no significant difference between the puree- and semisolid-consistency categories for the 7-month-old age group. 


\section{Discussion}

The purpose of this investigation was to quantify developmental changes in the temporal characteristics of chewing based on three-dimensional, optically based recordings of jaw movements. The results revealed both age- and consistency-related changes in chewing rate, sequence duration, and the estimated number of chewing cycles with consistency differences affecting masticatory timing in children as young as 7 months of age. The estimated number of chewing cycles decreased with age and varied, depending on consistency type. For example, the estimated number of chewing cycles in children as old as 35 months of age was larger than those of adults for certain consistency categories. This finding suggests that, although the basic coordinative organization for chewing is established early, some aspects of chewing development are protracted. The results also revealed that chewing sequences became shorter in duration with age and that chewing rate did not change appreciably with age. Taken as a whole, the findings from this study suggest that the different temporalchewing variables follow distinct developmental timelines and that the measures are consistency dependent even in children as young as 7 months of age.

\section{Kinematic Evidence of Increased Chewing Effectiveness With Age}

The decrease in the estimated number of chewing cycles with age is likely a result of the participants' experience with a variety of bolus types as well as their improved ability to efficiently detect consistency type and generate the appropriate amount of force and oromotor control to manipulate the food into a cohesive bolus (Wilson \& Green, 2009). The emergence of dentition was likely also a major contributing factor to the observed decrease in estimated number of chewing cycles with age. More specifically, Widmer (1992) reports that "by the age of 16 months the first primary molars attain occlusal contact" (p. 1252). Although there are no data at this specific age in the current investigation, one could speculate that the occlusal contact achieved by the molars provides greater occlusal shearing force, thereby improving chewing effectiveness. In general, the adults were more effective than several of the younger age groups at chewing the puree- and solid-consistency food, suggesting that, along with the advantage of dentition, improvements in oromotor control, interactions between the food and masticatory anatomy, and changes in sensorimotor awareness of bolus consistencies all play a role in the development of chewing effectiveness. Further work is needed to determine the age at which children become as effective as adults within certain consistency categories.
The current finding revealed an interaction between the estimated number of chewing cycles and age. More specifically, children as old as 35 months of age did not yet demonstrate an adultlike estimated number of chewing cycles for the most basic and earliest introduced consistency type-puree. This finding corroborates prior suggestions that, although the basic coordinative organization for chewing is established early in ontogeny, the emergence of specific aspects of chewing development follows a protracted trajectory. Studies on the development of speech movements have reported a similar finding (Smith \& Zelaznik, 2004).

In contrast, within the semisolid-consistency category, adultlike values for the estimated number of chewing cycles were achieved prior to 35 months of age. Gisel (1991) similarly reported delayed maturation for effective management of puree consistency and proposed that puree food may require greater oral motor skills than more viscous foodstuff. That is, more dense textures may potentially provide a more intense and richer supply of sensory feedback from oral receptors, which could serve to facilitate sensorimotor control of chewing movements.

\section{Chewing Sequence Duration Is Shorter for Adults Than for Children Regardless of Con- sistency Type}

The development of chewing was marked by a decrease in chewing sequence duration. One exception involved the 7-month-old children who appeared to chew the solid-consistency food for the same amount of time as the adult age group. However, this result should be viewed cautiously as the solid consistency data for the 7-month-old participants included only five chewing cycles across two participants; the remaining participants were not yet capable of managing a solid consistency bolus (see Table 4). In contrast, the adult data set for the solid-consistency category included 56 chewing sequences across all 12 participants.

Interestingly, the observed change in chewing sequence duration and not chewing rate suggests that adults have learned to produce a highly effective chewing cycle motion and, as a result, require fewer cycles per sequence for adequate bolus breakdown. This notion was further corroborated by our measure of the estimated number of chewing cycles (see Figure 5). Taken together, these results support earlier findings, which revealed that an essential aspect of chewing development was learning to accurately and efficiently scale jaw force and movement (Wilson \& Green, 2009). A reduction in movement variability and overshoot is a developmental phenomenon that has been documented across a variety of body systems, including precision grip (Forssberg, 
Eliasson, Kinoshita, Johansson, \& Westling, 1991), spatial aspects of chewing development (Wilson \& Green, 2009), speech development (Green, Moore, Higashikawa, \& Steeve, 2000; Green \& Nip, 2010; Smith \& Zelaznik, 2004), and reaching (Mathew \& Cook, 1990; Thelen, 1995). The decrease in chewing duration with age is likely a result of the increased ability to efficiently generate adequate occlusal force and scale movement to most effectively break down a food bolus.

\section{Upgrades in Consistency Affect Masticatory Kinematics Even in Very Young Children}

Relatively few studies have reported the effects of bolus consistency on chewing kinematics in children (Wilson \& Green, 2009). The age at which consistency effects are first observed may be an indicator of when children first acquire the sensory awareness of different foodstuff and/or develop the appropriate level of motor control to respond differentially to varying consistencies. We also acknowledge that the biomechanical properties of varying consistencies play a role in the development of jaw kinematics. The current results revealed that masticatory timing in children is affected by consistency changes, with some effects observed as early as 7 months of age. On the basis of visual observation of the chin, Gisel (1991) similarly observed consistency effects on chewing timing as young as 6 months of age. In contrast, in a prior investigation, consistency effects on spatial aspects of early chewing kinematics were not observed until 18 months of age (Wilson \& Green, 2009). Collectively, these findings suggest there is a different developmental trajectory for spatial and temporal aspects of chewing.

Although consistency effects on chewing rate varied with age, consistency effects on chewing sequence duration were constant across ages. That is, for all ages, (a) the sequence duration for puree-consistency food was shorter than the duration for semisolid-consistency food and (b) the sequence duration time was longest for solid-consistency food. Similarly, the estimated number of chewing cycles was significantly larger for the solid-consistency food than for the semisolid food regardless of age, and the estimated number of chewing cycles was significantly larger for the semisolid-consistency food than for the puree-consistency food for all ages studied, with the exception of the 7-month-old age group. Consistency, therefore, should be considered during the clinical evaluation of oral motor skills because temporal and spatial (Wilson \& Green, 2009) aspects of chewing, including chewing rate, chewing sequence duration, and the estimated number of chewing cycles, are influenced by bolus consistency.

\section{Chewing Rate Varied as a Function of Age and Consistency}

Major age-related changes in chewing rate were not observed with a few exceptions. More specifically, it was determined that chewing rate within the solidconsistency category was significantly faster in the 7-month-old children than in the 12-month-old children, and the beginner age group chewed the pureeconsistency food at a slightly slower rate than did the 7-month-old, 12-month-old, and adult participants. The latter finding was not unexpected because chewing was highly novel to the beginning chewers; however, the differences in average chewing rate for the puree-consistency food between the age groups were small, ranging between 0.15 and $0.27 \mathrm{~Hz}$. These kinematic-based findings corroborate previous findings that were based on visual observation of the chin (Gisel, 1988; Schwaab et al., 1986; Schwartz et al., 1984; Sheppard \& Mysak, 1984) and electromyography (Green et al., 1997; Steeve et al., 2008). The range for chewing frequency could have been considerably larger because adults are capable of producing jaw oscillations up to $6-8 \mathrm{~Hz}$.

\section{Speculation About the Central Mechanisms That Control Chewing Timing}

As noted previously, the findings from the current investigation and others suggest that the range for the average rate of chewing is relatively small (see Table 1). Interestingly, Gisel and colleagues reported that chewing rate was also not significantly different between children with Down syndrome and children who were typically developing (Gisel, Lange, \& Niman, 1984). The constancy of chewing rate to anatomic growth in typically developing children and children with neuromotor impairments related to Down syndrome suggests that the central mechanisms, such as the putative chewing CPG that regulate chewing timing, are very robust.

\section{Clinical Implications}

The quantitative information about the specific temporal aspects of early chewing development detailed in this investigation may be helpful in identifying and gauging the severity of early feeding disorders. For example, the results from this investigation, along with others, could be used to establish a developmental timeline highlighting specific expectations for each aspect of chewing performance. A child's performance could then be compared with the typical developmental progression to assist in identifying the nature and severity of specific masticatory deficits. Similarly, this timeline could be used as a reference for the creation of developmentally appropriate therapeutic approaches. 


\section{Experimental Limitations}

The purpose of this developmental investigation was to quantify the temporal characteristics of jaw motion for chewing. Regardless of age, the chewing behavior studied began at the point of maximal jaw closure after the food was placed in the oral cavity and ended at the swallow. We acknowledge that there are differences in the way the various age groups manage a bolus and that we captured the full spectrum of prechewing behaviors, but there is no definitive way to classify the prechewing behaviors other than to look at what the children do from a developmental perspective and determine how similar or dissimilar their behavior is in terms of adultlike chewing. It was in fact these developmental differences that we aimed to quantify.

Finally, although data are reported for all consistency categories within the 7-month-old age group (see Tables $3 \& 4$ ), only two of the 7-month-old children were capable of managing all three consistencies. We felt it important, however, to report what data were available for the 7-month-old participants, providing preliminary data that motivate more rigorous investigations of children at this age in future investigations. Further, because of the small number of participants, alpha adjustments were not performed because this approach is overly conservative; future work is necessary to corroborate the current findings.

\section{Conclusions}

The findings from this investigation indicate that both age- and consistency-related effects are evident in the development of masticatory timing. In general, it was determined that changes in consistency affect measures of masticatory timing in children as young as 7 months of age and that, while some aspects of chewing development are established early in ontogeny, other aspects are protracted beyond 35 months of age. It was also determined that chewing sequence duration decreased with age, as did the estimated number of chewing cycles for specific consistencies. Further, chewing rate varied as a function of age, although minimally. In the future, the quantitative information about the temporal aspects of early chewing development detailed in this investigation may be helpful for gauging the nature and severity of early feeding disorders and creating developmentally appropriate therapeutic approaches.

Acknowledgments - This project received funding and support from National Institute on Deafness and Other Communication Disorders Grants R03 DC004643, T32 DC05359, F31 DC006337, and R01 DC006463; National Institute of Child Health and Human Develop- ment Grant T32 HD07489; and a New Century Scholars Grant awarded to the first author by the American Speech-Language-Hearing Foundation. We would also like to acknowledge Lindsey Frederixon, Jimin Lee, Andrea Kettler, and Katherine Rogers for their assistance with data analysis; Daniel Bolt for statistical consultation; Chelsea Price for assistance with article preparation; Erin M. Major for her clinical expertise; and the children and their families for their participation.

\section{References}

Agrawal, K. R., \& Lucas, P. W. (2002). A review: Neural control of mastication in humans as influenced by food texture. Indian Journal of Dental Research, 13, 124-134.

Ahlgren, J. (1966). Mechanism of mastication: A quantitative cinematographic and electromyographic study of mastication movements in children, with special reference to occlusion of the teeth. Acta Odontologica Scandinavica, 24, 5-109.

Anderson, K., Throckmorton, G. S., Buschang, P. H., \& Hayasaki, H. (2002). The effect of bolus hardness on masticatory kinematics. Journal of Oral Rehabilitation, 29, 689-696.

Arizumi, K. (1989). Experimental studies concerning the effect of food consistency on masticatory movement in man. The Journal of the Japan Prosthodontic Society, 33, 1301-1312.

Arvedson, J. C. (1993). Oral-motor and feeding assessment. In J. C. Arvedson \& L. Brodsky (Eds.), Pediatric swallowing and feeding: Assessment and management (pp. 249-291). San Diego, CA: Singular.

Arvedson, J. C., \& Lefton-Greif, M. A. (1996). Anatomy, physiology, and development of feeding. Seminars in Speech and Language, 17, 261-268.

Arvedson, J. C., Rogers, B., \& Brodsky, L. (1993). Anatomy, embryology, and physiology. In J. C. Arvedson \& L. Brodsky (Eds.), Pediatric swallowing and feeding: Assessment and management (pp. 5-51). San Diego, CA: Singular.

Barlow, S. M., \& Estep, M. (2006). Central pattern generation and the motor infrastructure for suck, respiration, and speech. Journal of Communication Disorders, 39, 366-380.

Bosma, J. F. (1986). Development of feeding. Clinical Nutrition, 5, 210-218.

Chmielewski, W., Feine, J. S., Maskawi, K., \& Lund, J. P. (1994). Recording mandibular movements using chin markers [Abstract]. Journal of Dental Research, 73, 114.

Dellow, P. G., \& Lund, J. P. (1971). Evidence for central timing of rhythmical mastication. Journal of Physiology (London), 215, 1-13.

Filipic, S., \& Keros, J. (2002). Dynamic influence of food consistency on the masticatory motion. Journal of Oral Rehabilitation, 29, 492-496. 
Finan, D. S., \& Barlow, S. M. (1996). The actifier: A device for neurophysiological studies of orofacial control in human infants. Journal of Speech, Language, and Hearing Research, 39, 833-838.

Finan, D. S., \& Barlow, S. M. (1998). Intrinsic dynamics and mechanosensory modulation of non-nutritive sucking in human infants. Early Human Development, 52, 181-197.

Forssberg, H., Eliasson, A. C., Kinoshita, H., Johansson, R. S., \& Westling, G. (1991). Development of human precision grip: I. Basic coordination of force. Experimental Brain Research, 85, 451-457.

Gisel, E. G. (1988). Chewing cycles in 2- to 8-year-old normal children: A developmental profile. American Journal of Occupational Therapy, 42, 40-46.

Gisel, E. G. (1991). Effect of food texture on the development of chewing of children between six months and two years of age. Developmental Medicine and Child Neurology, 33, 69-79.

Gisel, E. G., Lange, L. J., \& Niman, C. W. (1984). Chewing cycles in 4- and 5-year-old Down's syndrome children: A comparison of eating efficacy with normals. American Journal of Occupational Therapy, 38, 666-670.

Green, J. R., Moore, C. A., Higashikawa, M., \& Steeve, R. W. (2000). The physiologic development of speech motor control: Lip and jaw coordination. Journal of Speech, Language, and Hearing Research, 43, 239-255.

Green, J. R., Moore, C. A., Ruark, J. L., Rodda, P. R., Morvee, W. T., \& VanWitzenburg, M. J. (1997). Development of chewing in children from 12 to 48 months: Longitudinal study of EMG patterns. Journal of Neurophysiology, 77, 2704-2716.

Green, J. R., \& Nip, I. S. B. (2010). Organization principles in the development of early speech: Catalysts, constraints, and synergy. In B. Maaseen \& P. H. H. M. van Lieshout (Eds.), Speech motor control: New developments in basic and applied research (pp. 171-188). New York, NY: Oxford University Press.

Green, J. R., Wilson, E. M., Wang, Y. T., \& Moore, C. A. (2007). Estimating mandibular motion based on chin surface targets during speech. Journal of Speech, Language, and Hearing Research, 50, 928-939.

Häggman-Henrikson, B., Eriksson, P. O., Nordh, E., \& Zafar, H. (1998). Evaluation of skin- versus teeth-attached markers in wireless optoelectronic recordings of chewing movements in man. Journal of Oral Rehabilitation, 25, 527-534.

Horio, T., \& Kawamura, Y. (1989). Effects of texture of food on chewing patterns in the human subject. Journal of Oral Rehabilitation, 16, 177-183.

Jemt, T., \& Hedgård, B. (1982). The relative movements of the chin and the mandible during chewing. Journal of Oral Rehabilitation, 9, 253.

Karkazis, H. C. (2002). EMG activity of the masseter muscle in implant supported overdenture wearers during chew- ing of hard and soft food. Journal of Oral Rehabilitation, 29, 986-991.

Karkazis, H. C., \& Kossioni, A. E. (1997). Re-examination of the surface EMG activity of the masseter muscle in young adults during chewing of two test foods. Journal of Oral Rehabilitation, 24, 216-223.

Karkazis, H. C., \& Kossioni, A. E. (1998). Surface EMG activity of the masseter muscle in denture wearers during chewing of hard and soft food. Journal of Oral Rehabilitation, $25,8-14$.

Lund, J. P. (1991). Mastication and its control by the brain stem. Critical Reviews in Oral Biology and Medicine, 2, 33-64.

Lund, J. P., Appenteng, K., \& Seguin, J. J. (1982). Analogies and common features in the speech and masticatory control systems. In S. Grillner, P. Lindblom, J. Lubker, \& A. Persson (Eds.), Speech motor control (pp. 231-245). New York, NY: Pergamon.

Lund, J. P., \& Kolta, A. (2006). Brainstem circuits that control mastication: Do they have anything to say during speech? Journal of Communication Disorders, 39, 381-390.

Lundeen, H. C., \& Gibbs, C. H. (1982). Advances in occlusion. Bristol, England: John Wright \& Sons.

Mathew, A., \& Cook, M. (1990). The control of reaching movements by young infants. Child Development, 61, 1238-1257.

Mioche, L., \& Peyron, M. A. (1995). Bite force displayed during assessment of hardness in various texture context. Archives of Oral Biology, 40, 415-423.

Morris, S. E., \& Klein, M. D. (2000). Pre-feeding skills: A comprehensive resource for feeding development (2nd ed.). Austin, TX: Pro-Ed.

Möller, E. (1966). The chewing apparatus: An electromyographic study of the action of the muscles of mastication and its correlation to facial morphology. Acta Physiologica Scandinavica, 69(Suppl.), 1-229.

National Dysphagia Diet Task Force. (2002). National dysphagia diet: Standardization for optimal care. Chicago, IL: American Dietetic Association.

Peyron, M. A., \& Mioche, L. (1994). Oral assessment of hardness between elastic and plastic products. Journal of Sensory Studies, 9, 223-236.

Peyron, M. A., Mioche, L., \& Culioli, J. (1994). Bite force and sample deformation during hardness assessment of viscoelastic models of foods. Journal of Texture Studies, 24, 59-76.

Peyron, M. A., Mioche, L., Renon, P., \& Abouelkaram, S. (1996). Masticatory jaw movement recordings: A new method to investigate food texture. Food Quality and Preference, 7, 229-237.

Pinder, G. L., \& Faherty, A. S. (1999). Issues in pediatric feeding and swallowing. In A. J. Caruso \& E. A. Strand (Eds.), Clinical management of motor speech disorders in children (pp. 281-318). New York, NY: Thieme. 
Pridham, K. A. (1990). Feeding behavior of 6- to 12-month-old infants: Assessment and sources of parental information. Journal of Pediatrics, 117, S174-S180.

Raudenbush, S. W., \& Bryk, A. S. (2002). Hierarchical linear models: Applications and data analysis methods. Thousand Oaks, CA: Sage.

Raudenbush, S. W., Bryk, A. S., \& Congdon, R. (2006). HLM 6.03: Hierarchical Linear and Nonlinear Modeling [Computer software]. Lincolnwood, IL: Scientific Software International.

Schwaab, L. M., Niman, C. W., \& Gisel, E. G. (1986). Comparison of chewing cycles in 2-, 3-, 4-, and 5-year-old normal children. American Journal of Occupational Therapy, 40, 40-43.

Schwartz, J. L., Niman, C. W., \& Gisel, E. G. (1984). Chewing cycles in 4- and 5-year-old normal children: An index of eating efficacy. American Journal of Occupational Therapy, 38, 171-175.

Sheppard, J. J., \& Mysak, E. D. (1984). Ontogeny of infantile oral reflexes and emerging chewing. Child Development, 55, 831-843.

Smith, A., \& Zelaznik, H. N. (2004). Development of functional synergies for speech motor coordination in childhood and adolescence. Developmental Psychobiology, 45, 22-33.

Squires, J., \& Bricker, D. (1999). Ages \& Stages Questionnaires: A parent-completed child-monitoring system. Baltimore, MD: Brookes.
Steeve, R. W., \& Moore, C. A. (2009). Mandibular motor control during the early development of speech and nonspeech behaviors. Journal of Speech, Language, and Hearing Research, 52, 1530-1554.

Steeve, R., Moore, C., Green, J., Reilly, K., \& Ruark McMurtrey, J. (2008). Babbling, chewing and sucking: Oromandibular coordination at 9 months. Journal of Speech, Language, and Hearing Research, 51, 1390-1404.

Steiner, J. E., Michman, J., \& Litman, A. (1974). Time sequence of the activity of the temporal and masseter muscles in healthy young human adults during habitual chewing of different test foods. Archives of Oral Biology, 19, 29-34.

Thelen, E. (1995). Motor development: A new synthesis. American Psychologist, 50, 79-95.

Widmer, R. P. (1992). The normal development of teeth. Australian Family Physician, 21, 1251-1261.

Wilson, E. M., \& Green, J. R. (2009). The development of jaw motion for mastication. Early Human Development, 85, 303-311.

Zafar, H., Nordh, E., \& Eriksson, P. O. (2002). Spatiotemporal consistency of human mandibular and head-neck movement trajectories during jaw opening-closing tasks. Experimental Brain Research, 146, 70-76. 